\title{
Emprendimiento, trabajos por cuenta propia y desempleo en la Región del Maule, Chile entre los años 2010-2016
}

Entrepreneurship, self-employment and unemployment in the Maule Region, Chile between the years $2010-2016$

Paola I. Escalona Sepúlveda Escuela de Contador Público y Auditor, Facultad de Economía y Negocios, Universidad de Talca. paolaescalona@gmail.com

Roberto M. Campos Troncoso Escuela de Contador Público y Auditor, Facultad de Economía y Negocios, Universidad de Talca. rcampos@utalca.cl

Felipe E. Arenas Torres Escuela de Contador Público y Auditor, Facultad de Economía y Negocios, Universidad de Talca. farenas@utalca.cl

\begin{abstract}
Resumen
Esta investigación correlaciona las variables: trabajos por cuenta propia, tasa de desempleo y la creación de microempresas en la región del Maule. Tomando como fuentes secundarias los datos entregados por el Instituto Nacional de Estadísticas y los datos de empresas pertenecientes a la base de datos del Servicio de Impuestos Internos de Chile. El propósito principal, fue determinar si existen correlaciones entre la tasa de desempleo, los trabajos por cuentas propia y la creación de microempresas. Según las cifras revisadas, en la Región del Maule, existe una relación inversa entre desempleo y trabajos por cuenta propia, no obstante no se puede afirmar lo mismo a nivel desagregado por ciudad, ni tampoco en la relación trabajos por cuenta propia y emprendimientos.
\end{abstract}

Palabras Claves: Cuenta propia, informalidad laboral, desempleo, emprendimiento.

\begin{abstract}
This research demonstrates the correlation between the following variables: self-employment, unemployment rate and the creation of micro-enterprises in the Maule region. Secondary sources were provided by the Instituto Nacional de Estadísticas and from companies whose data is stored with the Servicio de Impuestos Internos of Chile. The main purpose of this research, was to determine whether there are statistical correlations between the unemployment rate, self-employed jobs and the creation of micro-enterprises. According to the revised figures, in the Maule region, there is an inverse relationship between unemployment and self-employment, however the same cannot be said at the level disaggregated by city, nor in the relationship between self-employment and entrepreneurship.
\end{abstract}

Keywords: Own account, labor informality, unemployment, entrepreneurship. 


\section{Introducción}

La palabra emprendimiento se deriva de la expresión en francés "entrepeneur", cuyo significado es estar listo a tomar decisiones o a iniciar algo (Rodríguez, 2009). En los siglos XVII y XVIII, recibía el calificativo de emprendedor el arquitecto o constructor que llevaba a cabo la construcción de una obra. Sin embargo, Richard Cantillón (1680-1734), introduce el término entrepeneur, como el individuo que asume riesgos en condiciones de incertidumbre, con los fisiócratas franceses (Rodríguez, 2009).

Según Rodríguez (2009), el concepto de emprendedor define a dos tipos de personas: aquel guerrero arriesgado, cuyo reconocimiento es por sus características personales, o por el jefe de estado o reino que planea estrategias o políticas, para obtener objetivos y ser reconocido por sus funciones.

Al respecto y siguiendo la línea de Rodríguez (2009), la concepción de emprendedor se fundamenta en estas dos ideas. Sin embargo esta concepción ha sufrido cambios a través del tiempo, estableciendo que el emprendedor es aquella persona que "está en un estado de innovación permanente, altamente motivada y comprometida con una tarea, que reporta características de planeación y ejecución, propensaalriesgoyalavezesquivalacomprensión de sus propias dinámicas" (Rodríguez, 2009). En Latinoamérica, la importancia del emprendedor reside básicamente, en que su iniciativa empresarial permite la superación de la pobreza, $y$ es un mecanismo efectivo para lograr la movilidad generacional (Castellani y Lora, 2014). Además se ha probado, que en algunos casos los hijos de empresarios, alcancen un mayor nivel educacional, mejorando las perspectivas futuras de la nueva generación (Castellani y Lora, 2014).
Existen dos tipos de emprendimiento, el social y el comercial, los cuáles comparten una base conceptual, que es la creación de algo nuevo o la innovación en algo existente, y se diferencian básicamente en la finalidad lucrativa de quien lo realiza. Mientras que el emprendimiento social busca la creación de valor social, el emprendimiento comercial, busca la riqueza personal y de los accionistas. (Austin et al., 2006).

Poco se ha estudiado sobre el emprendimiento social, pero según Austin, Stevenson y WeiSkillern (2006), existen 4 dimensiones por las que nace el emprendimiento social que son:

1) Las fallas de mercado, es decir aquellos espacios no cubiertos por el emprendimiento comercial y abren brechas para la gestación de un emprendimiento social.

2) La misión que persigue, que como se mencionó anteriormente es la innovación social.

3) los recursos movilizados que es una de los elementos más diferenciadores con el emprendimiento comercial, dado que no existen compensaciones ni para los recursos humanos ni sobre el capital financiero invertido.

4) La medición del desempeño que presenta mayor complejidad que en un emprendimiento comercial, puesto que mide elementos de bienestar social, los que per ce son subjetivos.

En este sentido, el propósito de este artículo, está enfocado a estudiar una de las tantas aristas del emprendimiento comercial, tratando de determinar su relación con los trabajos por cuenta propia y en un segundo ámbito con la tasa de desempleo en la Región del Maule, Chile. 


\section{Marco Conceptual}

Es interesante observar que tanto la teoría de la firma como las teorías relacionadas con emprendimiento se han tratado de manera independiente, cuando el emprendedor es el individuo que combina, organiza y adquiere recursos heterogéneos en condiciones de incertidumbre (Klein, 2016), abriéndose un amplio espectro de investigación para definir en primer lugar, porqué surgen las oportunidades empresariales, segundo, porqué ciertos individuos descubren y explotan estas oportunidades y otros no, y tercero, porque existen diferentes modos para explotar dichas oportunidades (Klein, 2016).

Schumpeter (1911) pretende explicar la dinámica de una economía capitalista, partiendo de que la idea central de las empresas es reaccionar de manera creativa-adaptativa para obtener cuasirentas extraordinarias a partir de las innovaciones en los mercados, a este proceso Scumpeter lo denomina destrucción creativa, el cual es incentivado por el ingreso de nuevas firmas (emprendedores) (Barletta et al., 2014).

Así, países con niveles de desarrollo similares, persisten en diferencias en la iniciativa emprendedora, algunos estudiosos del tema sostienen que el PIB pér capita y la iniciativa emprendedora tiene una relación directamente proporcional, mientras que otros sostienen que es una relación indirecta. Por esto se cree que existen factores culturales que condicionan las iniciativas emprendedoras y que en el caso particular de Chile, el emprendimiento es impulsado por la valoración de crear una sociedad más igualitaria (Fernandez-Serrano y Liñan, 2014). Además, el emprendimiento está condicionado por las regulaciones estatales respecto del mismo, regulaciones en áreas tales como: gasto público, inversión, normativa laboral y prestaciones sociales para los desempleados (Alvarez, et al., 2014).

Según estudios del Global Entrepeneurship Monitor GEM, en 2017 en Chile se observó una alta valoración social a emprender, y cerca de un $40 \%$ de la población adulta, tiene la iniciativa de emprender, considerando el emprendimiento una opción de carrera deseable. Según el mismo reporte, los chilenos emprenden como una posibilidad de mejorar sus condiciones actuales, más que por la necesidad de emprender (Mandakivic y Serey, 2017).

Por otra parte, con respecto al financiamiento de los emprendimientos, se puede señalar que en Chile generalmente son financiados por los mismos emprendedores, quienes colocan los fondos personales como inversión inicial (Amorós et al., 2008). Según Amorós (2008), en Chile, no existe un desarrollo de fondos de inversión para financiar emprendimientos, aunque en el último tiempo, el estado a través de distintos programas, ha incentivado la creación de fondos de inversión temprana.

Al respecto, el trabajo de Barrera (2018), concluye sobre ciertos elementos claves a la hora de describir la innovación empresarial. Los empresarios que se auto perciben innovadores, sostienen que existen mayores barreras de entrada al negocio, ya que requieren más recursos en pos de implementar sus propuestas, además ellos creen que las condiciones económicas no los afectan, dadas sus capacidades individuales.

Asimismo, las mujeres emprendedoras son una proporción menor del género, por lo que obviamente se consideran innovadoras (Вагrera, 2018), según el GEM, la intención de emprendimiento femenino aumentó de un $10 \%$ a un 48\%, entre los años 2005 y 2015 (Montero et al., 2015). En el estudio de Вarrera (2018) también 
se concluye que la edad favorece la experiencia en los negocios, adoptándose a la vez menos riesgos de inversión y una menor tendencia a crear nuevas empresas o negocios. En este mismo estudio Barrera (2018), manifiesta que el nivel de educación fortalece la capacidad cognitiva, favoreciendo la eficacia, lo que permite adquirir competencias para buscar nuevas soluciones, generándose así una mayor autopercepción innovadora, en este mismo sentido en el año 2015 el $20 \%$ de las emprendedoras declara tener formación universitaria completa (Montero et al., 2015). Por último, la literatura sobre el emprendimiento, señala que las características emprendedoras tienen como fuente la capacidad empresarial, las características personales y la intuición, (Merigó y Peris-Ortiz, 2014) elementos que pueden servir раra la toma de decisiones.

La variable en estudio, trabajador(a) por cuenta propia en Chile, se define como "aquellos trabajadores que desempeñan su labor de manera autónoma e independiente, sin que exista una relación de dependencia con un empleador y que no registran renta en el Servicios de Impuestos Internos" (Subsecretaria de Previsión Social, 2018).

Al desglosar esta definición se puede rescatar dos elementos muy relevantes para entender qué se entiende por este tipo de trabajadores(as), primero se observa que son personas que no dependen de un empleador, es decir, no tienen una relación contractual con persona alguna o institución (Subsecretaria de Previsión Social, 2018), y en segundo lugar no emiten boleta de honorarios por los servicios prestados, sino más bien hacen un trabajo, sin ningún documento formal que valide la prestación dada, por ende dichas personas, son informales en el mercado laboral local, los que hace que su trabajo sea considerado de menor calidad, respecto de los asalariados, contando además, su vulnerabilidad, dado que no acceden a la red de protección social (Moyano et al., 2008)

Al respecto, las mayores tasas de informalidad laboral, están en los segmentos de mujeres, personas con bajos niveles educativos, jóvenes y mayores de 65 años. En estos segmentos la informalidad laboral es una opción, específicamente para las mujeres, representa una alternativa para compatibilizar trabajo y familia (Peticará y Celhay, 2010). Cuando se habla de calidad del empleo, no existe una única definición, aunque según la Organización Internacional del Trabajo, OIT, existen condiciones mínimas para entender la calidad laboral tales como: la existencia de un contrato laboral, una remuneración adecuada, la afiliación a la seguridad social y una jornada laboral justa, en otras palabras es la formalización de un trabajo (Jiménez y Páez, 2014).

En general los trabajos por cuenta propia son trabajos asalariados sin seguridad laboral, siendo ejecutados por individuos no profesionales y en algunos casos, tienen carácter temporal (Moyano et al., 2008). La particularidad del trabajo por cuenta propia es que tiene un comportamiento contra cíclico, es decir, que aumenta cuando hay una disminución del Producto Interno Bruto (PIB), no así el trabajo asalariado, aquel en donde existe una relación de dependencia con un empleador, cuyo comportamiento es pro cíclico, es decir que va en la misma dirección del crecimiento económico, situación observada en Chile los últimos 20 años (Szederkenyi y Vergara, 2017).

Según la caracterización de Szederkenyi y Vergara (2017), los trabajadores por cuenta propia, son aquellos trabajadores de servicios personales y hogares, y en agricultura sin diferencias significativas entre hombre y mujeres, aunque últimamente con preponderancia de hombres 
cuyo nivel educacional es básico y medio.

Siempre en el contexto de los empleos por cuenta propia, actualmente en Chile, el Instituto Nacional de Estadísticas realiza una encuesta de Microemprendimento, cuya $V$ versión, sacó resultados en enero de 2018. Esta encuesta de Microemprendimiento es aplicada cada dos años, y es un acuerdo suscrito entre la Subsecretaría de Economía y las Empresas de Menor Tamaño $y$ el Instituto Nacional de Estadísticas (INE). El énfasis de dicha encuesta, en su $V$ versión son las empresas de menor tamaño con 10 trabajadores o menos, enfocándose en una muestra representativa a nivel nacional y regional (Instituto Nacional de Estadísticas, 2018), denominando trabajadores por cuenta propia a aquellos que no tienen personal que trabaje en sus negocios.

Dicho lo anterior, pareciera haber una confusión de conceptos, puesto que el manual de dicha encuesta sostiene que puede ser considerado trabajador por cuenta propia aquel que esta formalizado pero no tiene trabajadores a su mando, lo que no se condice con lo declarado por la Subsecretaria de Previsión Social (2018).

Este artículo parte de la premisa, que los trabajos por cuenta propia, son potenciales emprendimientos y que a su vez disminuyen la tasa de desempleo, por lo tanto de alguna manera se está subestimando dicha tasa (Moyano et al., 2008), por lo anterior se pretende, determinar relaciones entre los trabajos por cuenta propia y los nuevos emprendimientos que año tras año, del periodo estudiado, se van creando en la Región del Maule.

Para realizar este trabajo se tomaron estadísticas de empresas del Servicio de Impuestos Internos (2016) y los resultados de la Encuesta Nacional de Empleo (Instituto Nacional de Estadísticas,
2010), cuyo propósito es medir el desempleo por trimestre móvil y que además reporta los trabajos por cuenta propia, junto con otros compendios estadísticos de INE.

En esencia este artículo está dirigido a:

1.-Hacer una descripción estadística de los llamados empleos por cuenta propia y las microempresas de la Región del Maule, entre los años 2010 al 2016, inclusive. No es recomendable hacer mediciones anteriores al 2010, pues antes el INE empleaba otra metodología en las mediciones del mercado laboral.

2.- Describir el comportamiento de la tasa de desempleo en la Región del Maule desde el año 2010 hasta el año 2016.

3.- Relacionar las variables antes descritas, de manera de hacer un análisis comparativo de sus respectivos comportamientos, calculando correlaciones estadísticas entre ellas.

4.- Analizar el comportamiento y correlaciones de las estadísticas de desempleo, trabajos por cuenta propia y la cantidad de las microempresas, entre los años 2010 y 2016, de los conglomerados urbanos de la Región del Maule (Talca, Curicó y Linares).

5.- Determinar correlaciones entre las tres variables antes descritas tanto a nivel local como regional.

\section{Materiales y Métodos}

Este trabajo es de tipo descriptivo cuantitativo y pretender describir el comportamiento de los últimos 7 años (2010-2016), con los que se cuenta información sobre los trabajadores por cuenta propia, la tasa de desempleo y 
los emprendimientos de la Región del Maule, Chile. En primera instancia busca correlacionar las tendencias de las variables antes definidas, mostrando las variaciones de las tres y relacionando las series antes mencionadas (Bernal, 2010).

La metodología utilizada en la confección de este trabajo es un análisis longitudinal entre la tasa de desempleo de la región del Maule y la evolución de los trabajos por cuenta propia, analizando a la par, la formalización de empresas según los registros del Servicio de Impuestos Internos (SII), tomando como base conceptual lo que señalan distintos autores hispanoamericanos respecto de los trabajos por cuenta propia (Bernal , 2010).

Para el desarrollo de este trabajo se usaron estadísticas de la Encuesta Nacional de Empleos (ENE) del Instituto Nacional de Estadísticas, la cual es aplicada por el INE todos los trimestres móviles. Se toma desde el 2010, porque a partir de ese año hubo un cambio metodológico en la aplicación de ésta, por lo cual los resultados de los años anteriores no son homologables. Рara efectos de este trabajo, las variables que se ocuparon fueron las tasas de desocupación o desempleo provistas por el INE y la variable que apela a los llamados empleos por cuenta propia, que según la encuesta, son aquellas personas que se autoemplean, pero no generan empleos para terceros.

Por ende, se buscó comparar los resultados de la ENE con las estadísticas más recientes sobre empresas, las que fueron tomadas de las bases de datos del Servicio de Impuestos Internos. Entre las estadísticas procesadas por el INE en la Encuesta Nacional de Empleos, destacan las tasas de desocupación de las ciudades más importantes del país, que para efecto de la Región del Maule son 3, Curicó, Talca y Linares. Dado el criterio de importancia impuesto por el INE, se procesan datos de empresas para estas ciudades. Por último, los datos fueron procesados con STATA 14, SPSS 23 y con EXCEL 2016.

\section{Desarrollo}

La Región del Maule se encuentra ubicada a 271 $\mathrm{Km}$. al sur de Santiago, la capital de Chile. Al norte limita con la región del Libertador General Bernardo O'Higgins, al sur con la región del Bío Bío, al oeste con el Océano Pacífico y al este con el límite internacional de la República de Argentina, tiene una superficie de $30.296,10 \mathrm{Km}$. cuadrados, consta de 4 provincias: Curicó, Talca, Linares y Cauquenes, entre las comunas más grandes destacan: Parral y Molina. (Biblioteca del Congreso Nacional de Chile, s.f.)

Según el CENSO Agropecuario 2007, está Región concentra el $54 \%$ del total de las exportaciones forestales y agropecuarias, junto a las regiones del Bío-Bío y la Araucanía, otro dato relevante según el CENSO Agropecuario, la Región del Maule concentra el $17,6 \%$ de las plantaciones forestales del país (Instituto Nacional de Estadísticas, 2007).

Lo anteriormente descrito, da luces de la estructura productiva de la Región del Maule, que preferentemente es agrícola, puesto que cuenta con un clima mediterráneo cálido y subhúmedo y en donde es posible encontrar manifestaciones y tradiciones propias del campo (Biblioteca del Congreso Nacional de Chile, s.f.). Con la reforma agraria de la década de los 60, el Maule pasó de una producción agrícola de grandes latifundios a una economía agraria de mercado, dicha reforma agraria tenía como consigna que "la tierra es para quien la trabaja", deshaciendo los grandes hacendados, que eran símbolo de poder para sus poseedores, y redistribuyendo la tierra entre 
los trabajadores agrícolas (Bellisario, 2013). Actualmente, el Maule cuenta con alrededor de 2 millones de hectáreas entre los distintos cultivos (semilleros, viveros, viñas, plantaciones forestales, frutales, flores, hortalizas, cereales, leguminosas y tubérculos).

En promedio, los ocupados en el Maule, entre los años 2010-2017 fueron 443 mil personas aproximadamente, de las cuales un $27 \%$ trabajan en actividades agrícolas, un $18 \%$ en actividades de comercio y un $10 \%$ en industrias manufactureras, mientras que el $45 \%$ restante se desempeña en actividades de construcción, enseñanza, administración pública, servicios bancarios, etc. (Instituto Nacional de Estadísticas, 2010).

El Gráfico 1 muestra la evolución de las tasas de desempleo y los empleos por cuenta propia de la Región del Maule, donde se pueden hacer dos observaciones sobre el comportamiento de ambas variables, la tasa de desempleo regional es oscilante, y tiene que ver con los ciclos agrícolas, donde se aprecia que en los meses de primavera y verano, la tasa de desocupación cae, puesto que más se emplean en las labores agrícolas. Mientras que en los meses de otoño comienza aumentar, logrando su peak en los meses de invierno, la tendencia de la tasa de desempleo, es a la baja, en los últimos 4 años.

Por otro lado al observar la representación de los trabajos por cuenta propia, tiende a ser estable a lo largo del tiempo, sin embargo, tiene una tendencia creciente.

La covarianza entre ambas variables y la correlación de Pearson dan cuenta de la relación indirecta que existe entre la tasa de desocupación y los trabajadores por cuenta propia, en palabras sencillas, cuando aumenta la tasa de desocupación disminuyen los trabajos por cuenta propia en la Región del Maule, y viceversa, correlación que se afirma con un test de hipótesis.

\section{Gráfico 1: Evolución de la tasa de desocupación y trabajadores por Cuenta Propia entre los años}

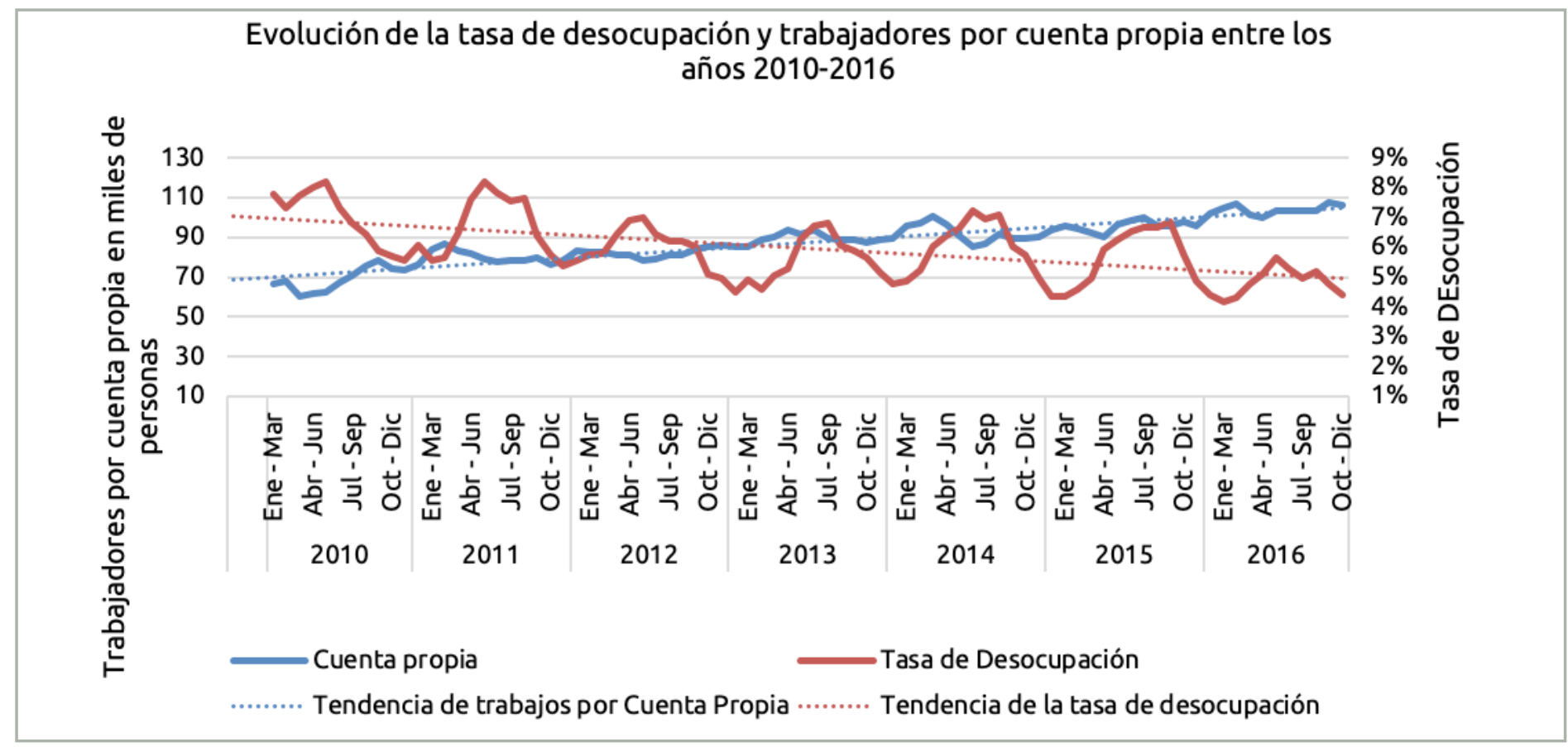

Fuente: Elaboración propia con datos tomados de www.ine.cl. 
Para entender el Gráfico 2, se debe saber cómo clasifica el Servicio de Impuestos Internos (SII) las empresas en Chile. Para el SII, existen 4 grandes clasificaciones de las empresas; grandes empresas, medianas, pequeñas y microempresas, este criterio está supeditado a las ventas en UF
[1] de cada empresa, a su vez, la clasificación de microempresa se subdivide en tres categorías, la pequeña empresa en 3 categorías, la mediana empresa, en 2 categorías y la gran empresa en 4 categorías. El cuadro $\mathrm{N}^{\circ} 1$ resume la clasificación:

\section{Cuadro N¹: Clasificación de las empresas según el Servicio de Impuestos Internos (SII).}

\begin{tabular}{|c|c|c|}
\hline Tamaño de Empresas. & Tipo & Rango \\
\hline \multirow[t]{3}{*}{ Microempresa } & Tipo 1 & 0,01 a 200 UF \\
\hline & Tipo 2 & 200,01 a 600 UF \\
\hline & Tipo 3 & 600,01 a 2.400 UF \\
\hline \multirow[t]{3}{*}{ Pequeña } & Tipo 1 & $2.400,01$ a 5.000 UF \\
\hline & Tipo 2 & $5.000,01$ a 10.000 UF \\
\hline & Tipo 3 & $10.000,01$ a 25.000 UF \\
\hline \multirow[t]{2}{*}{ Mediana } & Tipo 1 & $25.000,01$ a 50.000 UF \\
\hline & Tipo 2 & $50.000,01$ a 100.000 UF \\
\hline \multirow[t]{4}{*}{ Grande } & Tipo 1 & $100.000,01$ a 200.000 UF \\
\hline & Tipo 2 & $200.000,01$ a 600.000 UF \\
\hline & Tipo 3 & $\begin{array}{l}600.000,01 \text { a } 1.000 .000 \\
\text { UF }\end{array}$ \\
\hline & Tipo 4 & Más de 1.000.000 UF \\
\hline
\end{tabular}

Fuente: Elaboración propia, con datos tomados de www.sii.cl

Dada la especificación anterior, al observar el número de microempresas maulinas registradas en el SII, existe una tendencia creciente a la creación de éstas, en sus distintos tipos, sin embargo, la mayor tasa de crecimiento en la creación, se observa entre los años 2013 y
2014 , con una tasa de crecimiento de un $2 \%$ y un $3 \%$, respectivamente. La tasa de crecimiento promedio en la creación de microempresas en el Maule, es de un 1,5\% respecto del año anterior. 


\section{Gráfico 2: Microempresas en la Región del Maule entre los años 2010-2016}

Microempresas en la Región del Maule entre los años 2010-2016

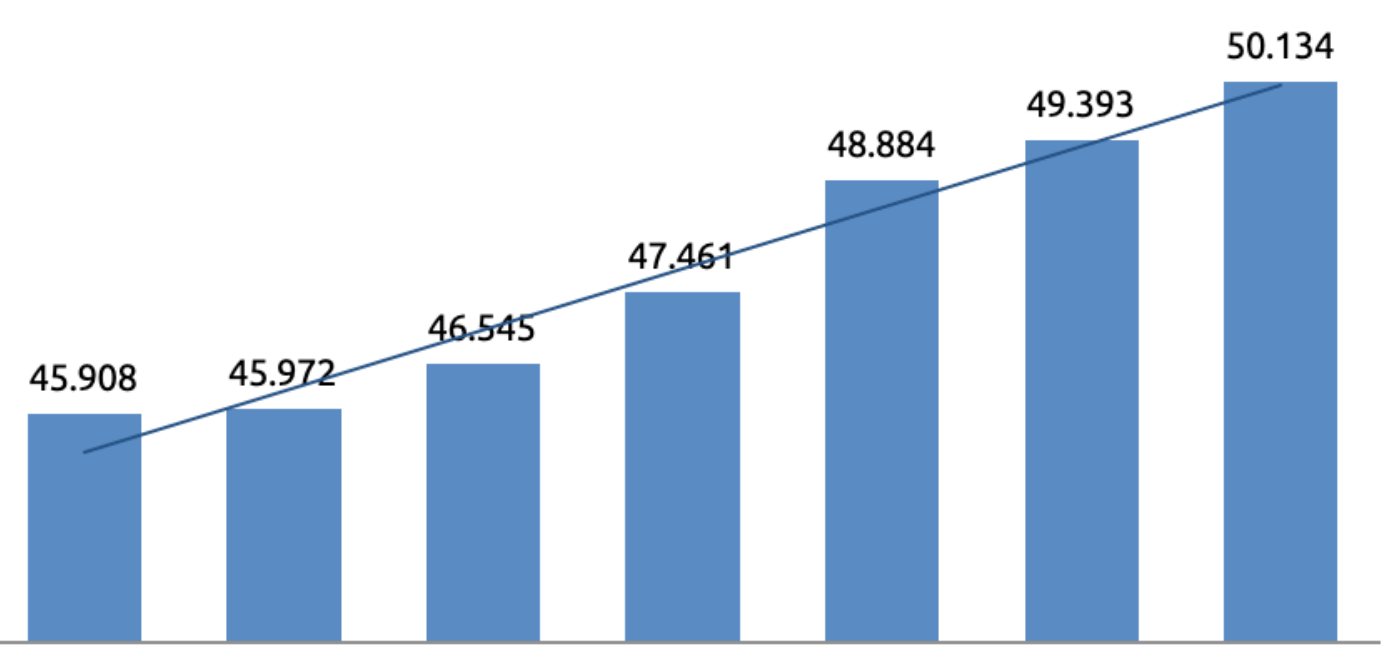

Total 2010 Total 2011 Total 2012 Total 2013 Total 2014 Total 2015 Total 2016

Fuente: Elaboración propia con datos tomados del www.sii.cl

Otros aspectos que parecen necesarios comparar, es la correlación existente entre los trabajadores por cuenta propia y los microempresarios en el Maule. Como se aprecia, la creación de microempresas entre los años 2010 al 2016, tendió a ser estable, alcanzando una mayor tasa de crecimiento en el año 2013, un 3\%, y como se señaló en el párrafo anterior, una tasa de
$1,5 \%$ de crecimiento promedio. Por otro lado los trabajadores por cuenta propia maulinos, alcanzaron su mayor tasa de crecimiento en el mismo 2013 con una tasa de 7,9\%, seguida por un crecimiento de $7,8 \%$ en el año 2016 . La tasa promedio de crecimiento de los trabajadores por cuenta propia fue de un, $7,3 \%$ en los años estudiados. 


\section{Gráfico 3: Comparación entre la evolución de Microempresas y la evolución de Trabajadores por Cuenta Propia entre los años 2010-2016.}

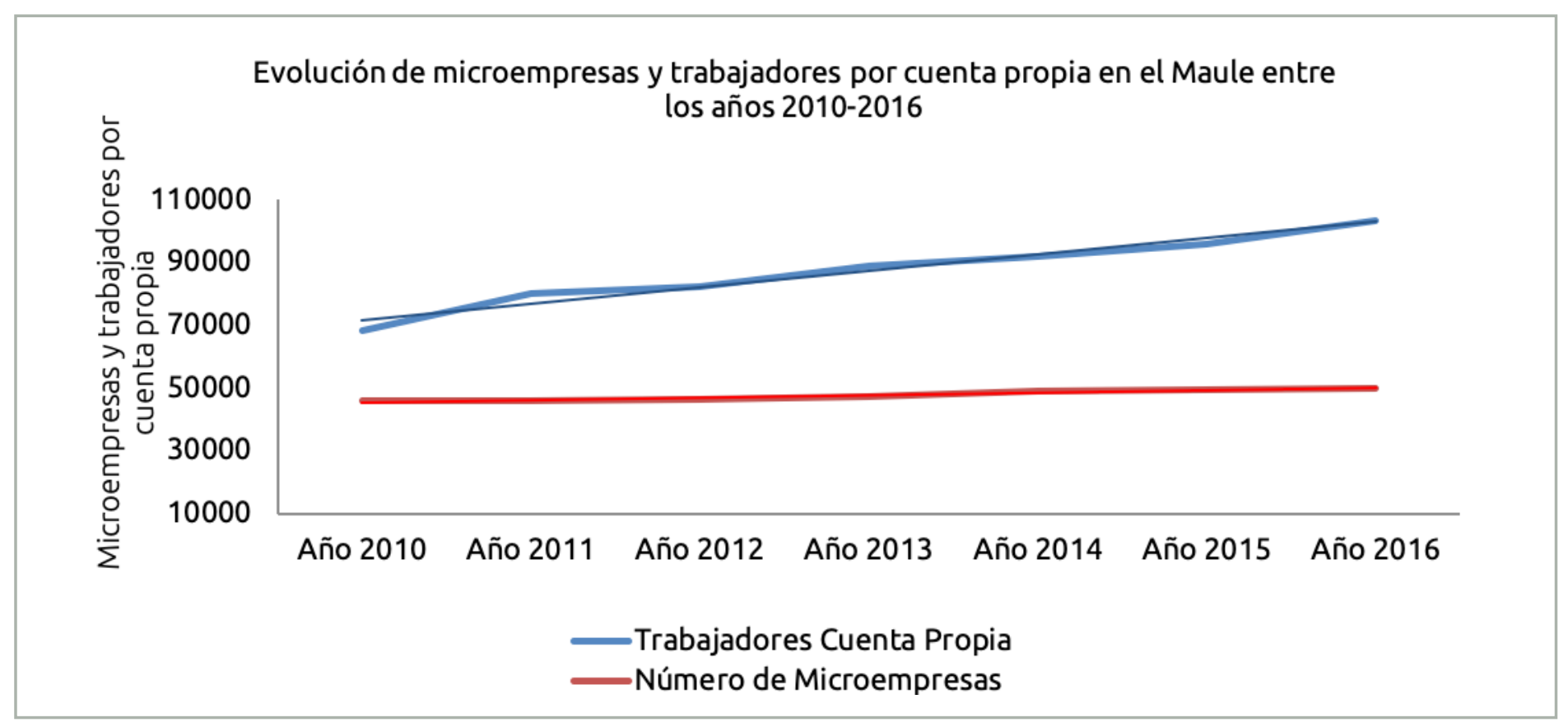

Fuente: Elaboración propia con datos tomados de www.ine.cl y www.sii.cl.

Es relevante también analizar en detalles cada una de las ciudades más importantes que componen la Región del Maule, en las cuales destacan, Curicó, Talca y Linares, la importancia de analizar estas ciudades viene dada porque estas son los tres conglomerados urbanos más grandes de la Región, cuya población es de alrededor de 100 mil habitantes y más.

La información procesada para este estudio fue obtenida desde las estadísticas de la Encuesta Nacional de Empleo, la cual entrega resultados significativos a nivel Nacional, Regional, Provincial y de las grandes ciudades de cada Región, (Instituto Nacional de Estadísticas, 2018). Estas ciudades, están dispersas a lo largo de la Región, con ciertas particularidades en sus matrices productivas.
Al comparar las 3 ciudades, se observa que el patrón del comportamiento de las tasas de desempleo, es similar para las tres, decrece en las primaveras y en los veranos y aumenta en los inviernos. La mayor variabilidad del empleo/ desempleo en los últimos 7 años, es en la ciudad de Talca, mientras que la menor, es en la ciudad de Linares, el desempleo promedio mayor es en Linares, mientras que el más bajo en Curicó; además a lo largo de los años analizados se tiene que la tasa mínima de desempleo la ostenta Talca, con un $2,6 \%$ en el trimestre móvil Diciembre de 2014 a Febrero de 2015, mientras que la más alta fue en Linares, de un $13,6 \%$ en el trimestre Julio-Septiembre de 2011. El comportamiento comparado de las tasas de desempleo se puede ver en el Gráfico 4: 


\section{Gráfico 4: Comparación de evolución de las tasas de desempleo entre las ciudades de Curicó, Talca y Linares de la Región del Maule.}

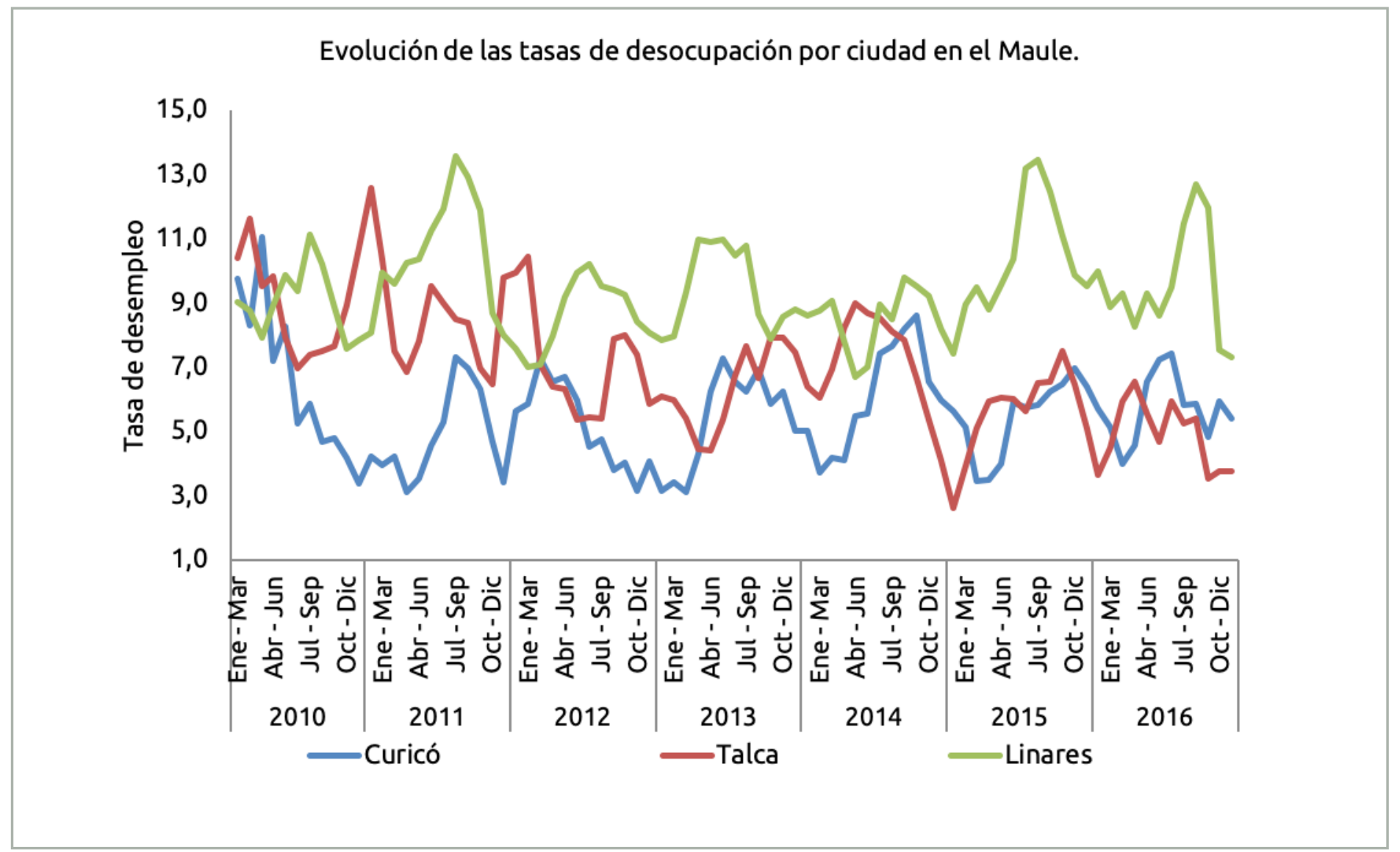

Fuente: Encuesta Nacional de Empleo, INE.

\section{Curicó}

La ciudad de Curicó es el mayor centro urbano por el norte de la Región del Maule, y según la actualización del Censo 2017 dicha comuna cuenta con 149.136 habitantes.

El Gráfico 5 muestra los trabajos por cuenta propia y la tasa de desempleo. Al analizar las hipótesis de correlación, no existe significancia estadística que evidencie correlación entre ambas variables. Un elemento importante a destacar sobre los empleos por cuenta propia, es que estos se acrecentan en los meses de invierno, lo que de alguna manera es intuitivo, puesto que en los meses de verano los maulinos trabajan en forma dependiente en las faenas agrícolas, no obstante se autoemplean en los meses de invierno. Así al observar las cifras se da cuenta que en los trimestres abril-junio y julio-septiembre, los empleos por cuenta propia subieron, con excepción en el año 2011, en donde el alza se observa en el trimestre OctubreDiciembre. 
Gráfico 5: Comparativo entre tasa de desempleo y trabajos por cuenta propia en la ciudad de Curicó, entre los años 2010-2016.

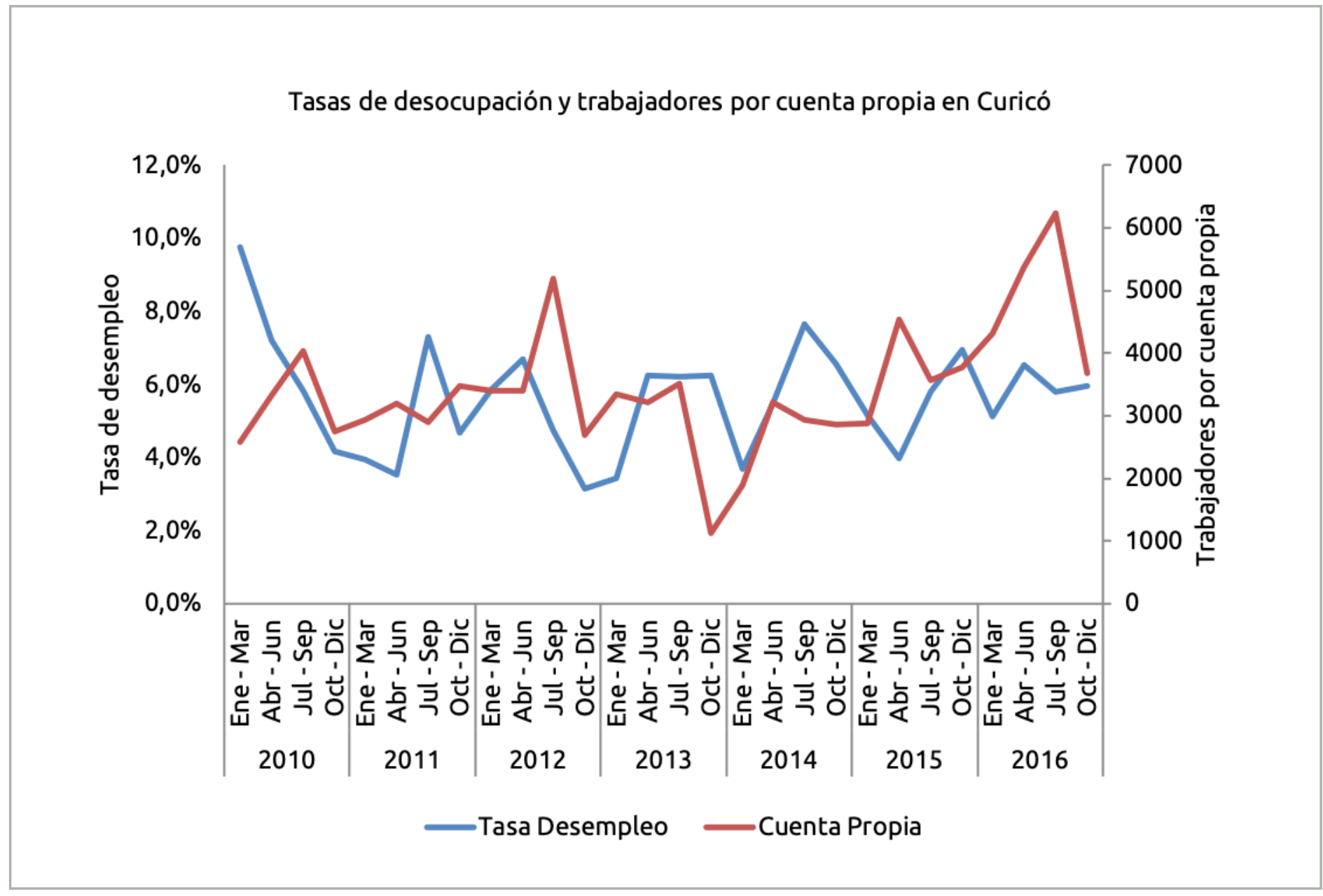

Fuente: Elaboración propia con datos tomados de la Encuesta Nacional de Empleo, www.ine.cl.

Si se comparan las estadísticas de la creación de microempresas del Servicio de Impuestos Internos con las cifras entregadas por la Encuesta Nacional de Empleos del INE, en los años estudiados se tiene que en Curicó, existe una brecha promedio de aproximadamente unas 3000 microempresas creadas versus los empleos por cuenta propia. Además se evidencia mediante el gráfico, que los empleos por cuenta propia crecieron entre los años 2015 y 2016, entre los mismos periodos en que la tasa de desempleo sufre una leve alza. Las cifras expuestas, evidencian correlaciones a un $5 \%$ de significancia estadística. 
Gráfico 6: Comparativo entre microempresas y trabajos por cuenta propia en la ciudad de Curicó, entre los años 2010 y 2016.

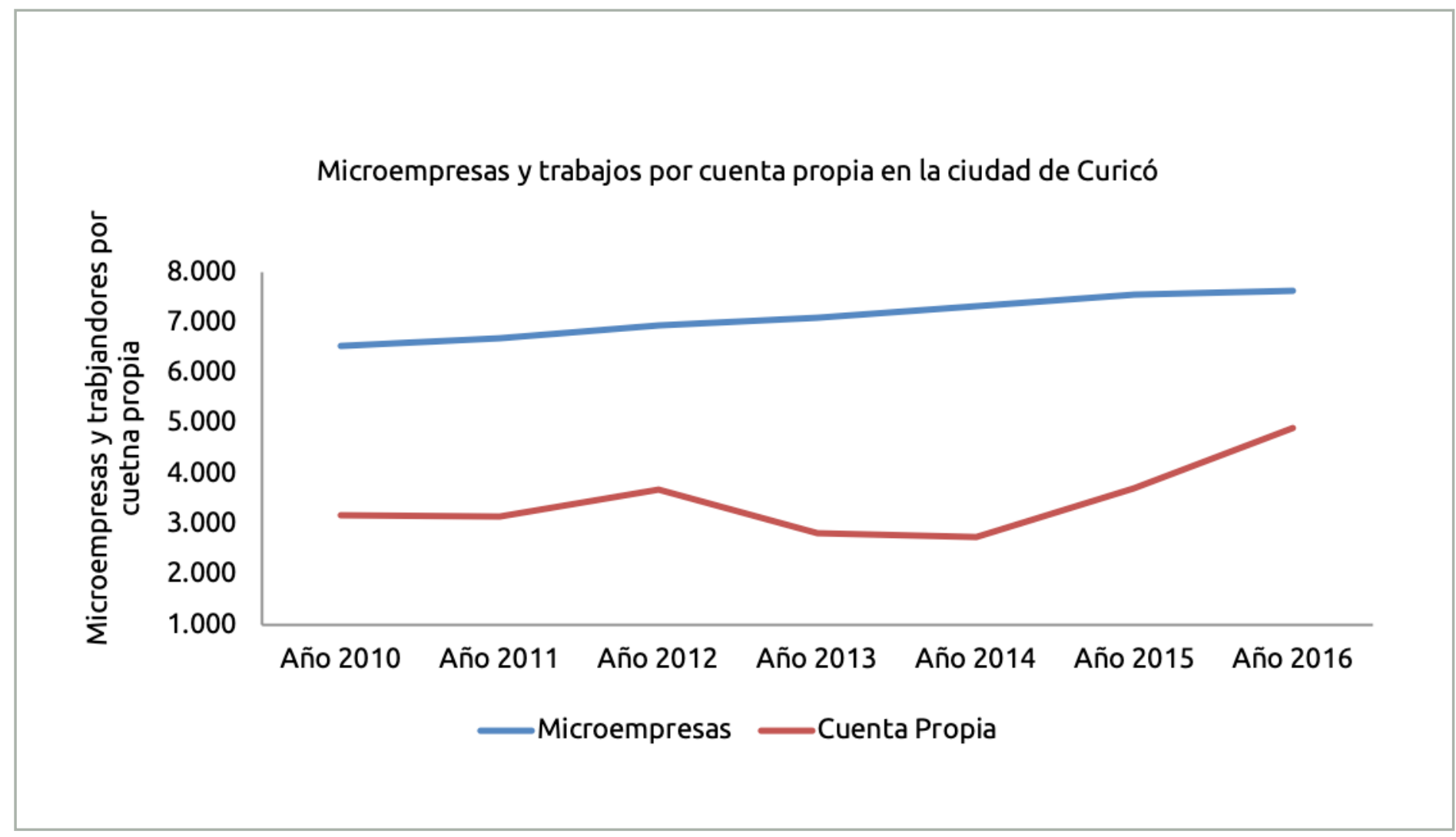

Fuente: Elaboración propia con datos tomados de www.ine.cl y www.sii.cl

\section{Talca}

De los centros urbanos de la Región del Maule, la ciudad de Talca es la que concentra el mayor porcentaje de la población, cuya comuna cuenta con 220.357 habitantes según la actualización del Censo 2017 (Censo, 2017). La ciudad de Talca, concentra todos los servicios, tanto públicos como privados de la región, dado su status de Capital Regional.
Como es típico de la Región del Maule, Talca también muestra un comportamiento oscilante en su tasa de desempleo y los empleos por cuenta propia, situación común en toda la Región, la explicación es básicamente la misma, la condición agrícola de la región, sin embargo al observar las estadísticas se deben resaltar ciertos comportamientos, el primero los empleos por cuenta propia tienen un aumento en los trimestre de verano, Octubre-Diciembre 
o Enero-Marzo, exceptuándose el año 2010, el cual se puede considerar un año atípico, dado el terremoto que aquejó la zona.

Por otro lado, al observar las tasas de desempleo, se observa que la menor tasa en los periodos estudiados no fueron necesariamente en los veranos, sino que se dieron con ciertas mixturas; en los años 2011, 2014, 2015 y 2016, las menores tasas de desempleo fueron en los trimestres de veranos, Octubre-Diciembre o Enero-Marzo, mientras que en los años 2010, 2011, 2013 las menores tasas se suscitaron en los trimestres de otoño e invierno, Abril-Junio o Julio-Septiembre. Este hecho puede tener varias explicaciones, una de ellas puede ser que Talca es una ciudad de servicios preferentemente, que si bien es cierto está en una región agrícola, pero su producción se concentra en Comercio y Servicios Personales, los que mayoritariamente se explica por su condición de capital regional.

Las series mostradas en el gráfico 7, no presentan una correlación estadística significativa.

Gráfico 7: Comparativo entre tasa de desempleo y trabajos por cuenta propia en la ciudad de Talca, entre los años 2010-2016.

Tasa de desocupación y trabajos por cuenta propia en la ciudad de Talca

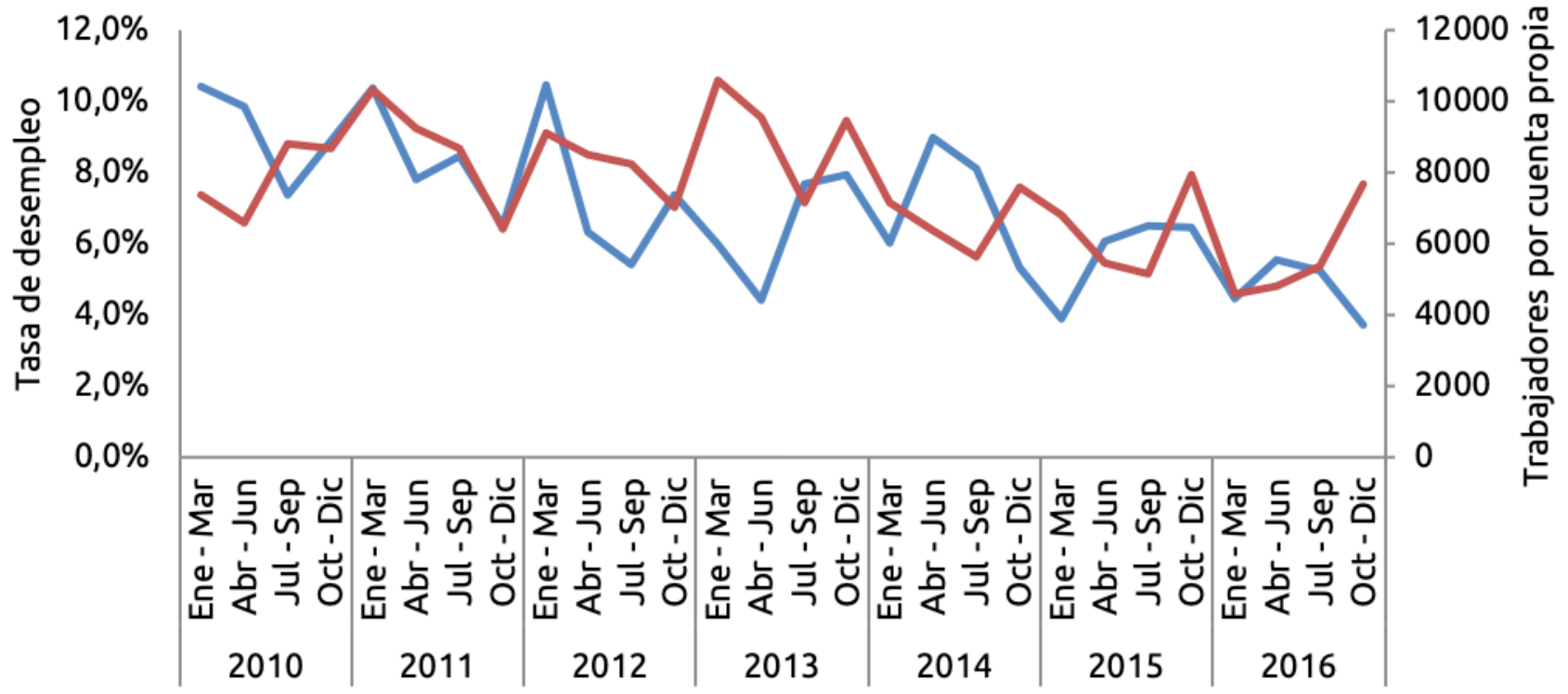

Tasa de desempleo Cuenta Propia 
El Gráfico 8 hace la comparación entre las microempresas y los trabajos por cuenta propia en Talca, la primera afirmación que se puede realizar es que no se evidencia una correlación entre ambas series, por lo que no se podría afirmar que los trabajos por cuenta propia se convierten en microempresas, lo que precisamente trata de testear este trabajo. Es difícil tener certeza de los datos, puesto que la Encuesta Nacional de Empleo, no pregunta sobre la formalización de los trabajos por cuenta propia a través del tiempo.

La brecha promedio entre la creación de microempresas y empleos por cuenta propia es de 2.140 emprendedores, pero al observar el gráfico, se aprecia que esta brecha se agudiza a partir del año 2014.

\section{Gráfico 8: Comparativo entre microempresas y trabajos por cuenta propia en la ciudad de Talca, entre los años 2010 y 2016.}

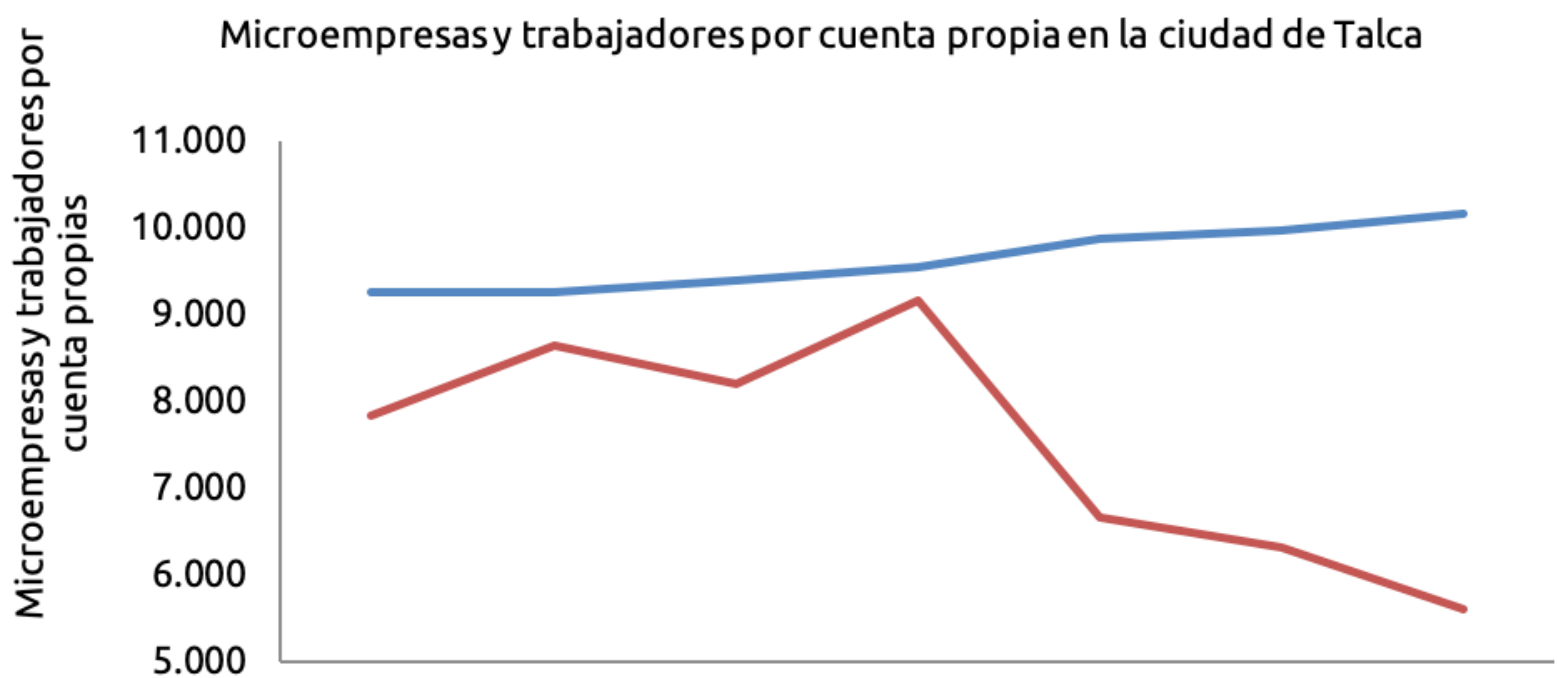

Año 2010 Año 2011 Año 2012 Año 2013 Año 2014 Año 2015 Año 2016

- Microempresas —Cuenta Propia

Fuente: Elaboración propia con datos tomados de www.ine.cl y www.sii.cl . 


\section{Linares}

La comuna de Linares, cuenta con 93.602 habitantes (Censo, 2017), de las 3 ciudades analizadas por la Encuesta Nacional de Empleos, es la más pequeña, y abunda en predios agrícolas y hacendados.

El Gráfico 9, hace la comparación de la tasa de desempleo y los trabajos por cuenta propia de dicha ciudad. Los trabajos por cuenta propia tienen su mayor apogeo en los meses de invierno, abril-junio y julio-septiembre, en los años 2010, 2013, 2014, 2015 sin embargo, en los trimestres de verano es cuando existen mayores empleos por cuenta propia, en los años 2011, 2012 y 2016. La tasa de desempleo más alta en esta ciudad fue en el trimestre julio-septiembre de 2011 y cuando más florecieron los empleos por cuenta propia fue en el trimestre Octubre-Diciembre de 2016, por otra parte la menor tasa de desempleo se dio en el trimestre Abril-Junio de 2014.

Si se analiza la relación entre ambas variables, no existe evidencia de la correlación entre ambas variables, entonces se puede afirmar que el coeficiente de correlación es cero.

\section{Gráfico 9: Comparativo entre tasa de desempleo y trabajos por cuenta propia en la ciudad de Linares, entre los años 2010-2016.}

Tasa de desocupación y trabajadores por cuenta propia en la ciudad de Linares

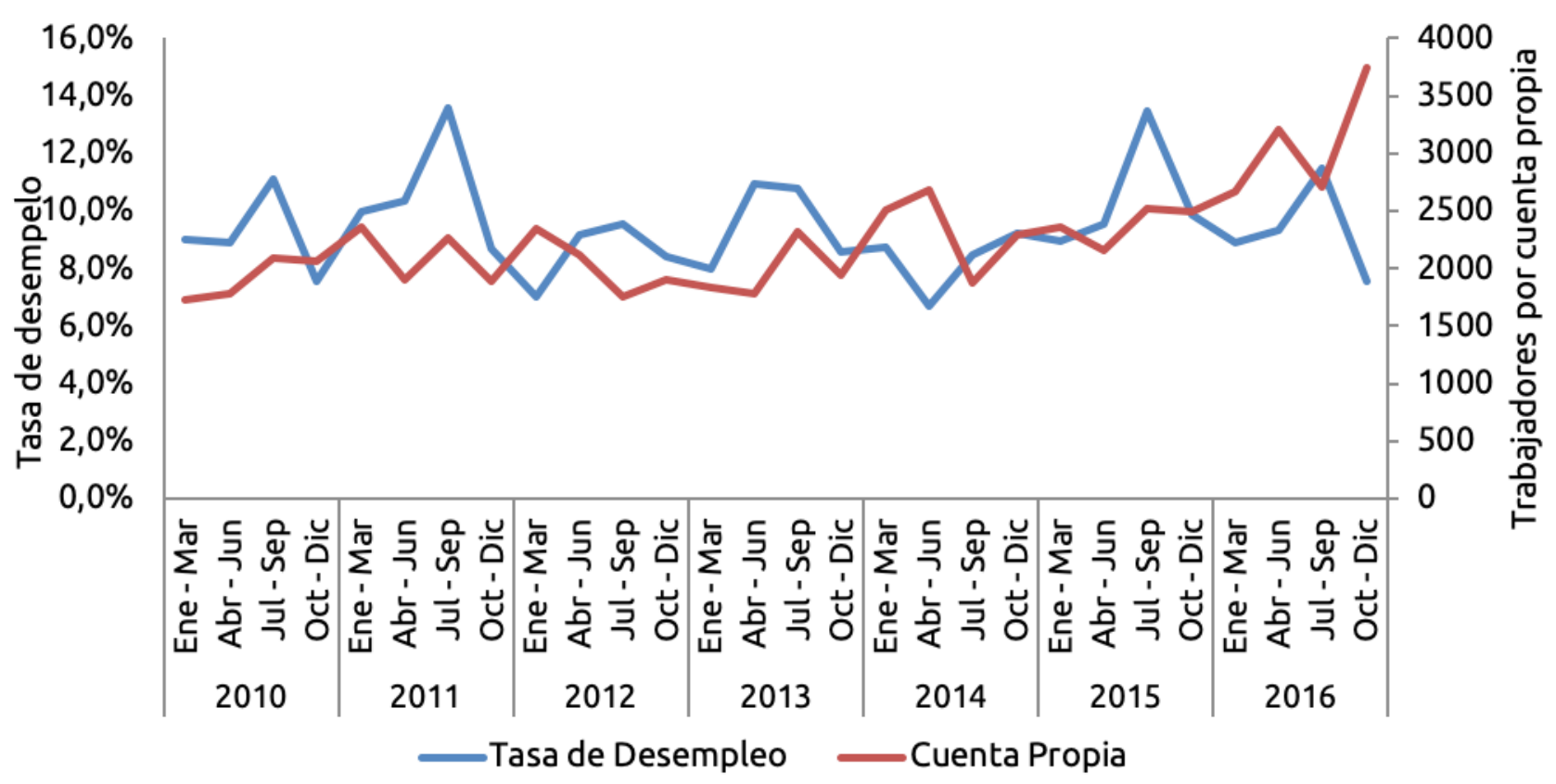


Según el Gráfico 10, existe una brecha de 2.047 emprendedores promedios entre los empleos por cuenta propia y las microempresas formalizadas en la ciudad de Linares, entre los años 2010 y 2016, evidenciándose que ambas variables están correlacionadas, con un nivel de significancia del $1 \%$. A finales del año 2016, la brecha entre los empleos por cuenta propia y la creación de microempresas se hace menor.

Gráfico 10: Comparativo entre microempresas y trabajos por cuenta propia en la ciudad de Talca, entre los años 2010 y 2016.

Microempresas y trabajadores por cuenta propia en la ciudad de Linares

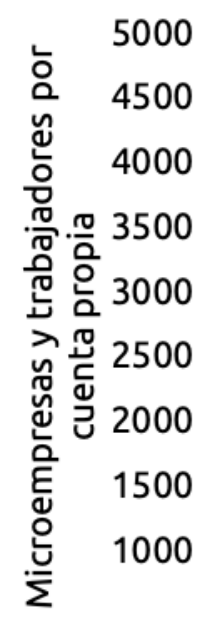

Año 2010

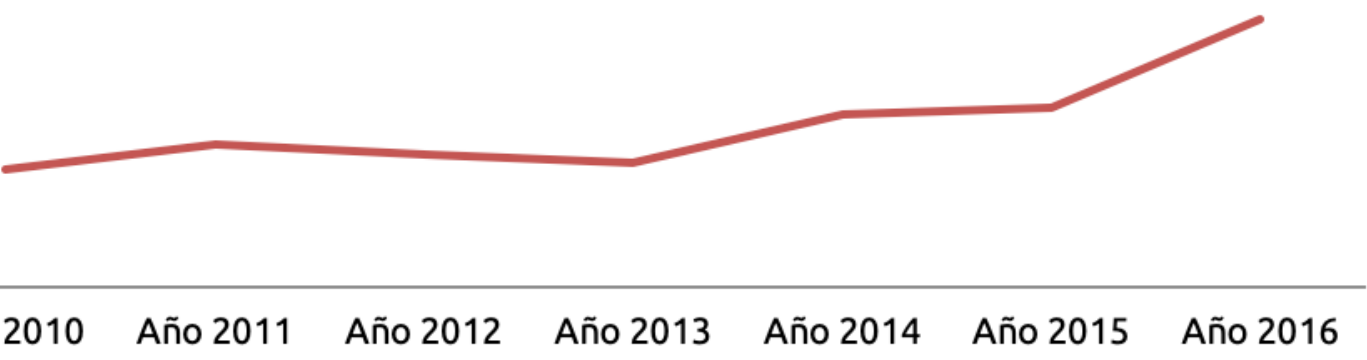

Microempresas

Cuenta Propia

Fuente: Elaboración propia con datos tomados de www.ine.cl y www.sii.cl . 


\section{Reflexiones finales}

Al observar las cifras regionales, se evidencia que existe una disminución en el desempleo cuando aumentan los trabajos por cuenta propia, lo que no queda claro de este hecho es qué variable afecta a la otra, sin embargo vale la pena preguntarse, cuántos de estos trabajos por cuenta propia se transforman en emprendimientos, y más aún, cuántos de estos perduran en el tiempo.

Cuando se comienza a profundizar en las estadísticas por ciudad, hay dos elementos claves; las grandes áreas urbanas no son fuentes de los trabajos por cuenta propia, sino que estos provienen de las ciudades con menos de los 90.000 habitantes y de los poblados de la región, junto con ello también se puede afirmar, que no existe una correlación clara entre el los empleos por cuenta propia y el desempleo en las grandes urbes, es más, en algunos momentos pareciera, que a mayor empleo, más trabajos por cuenta propia, cosa que no se evidencia en los agregados regionales.

De lo anterior subyacen las siguientes conclusiones, los trabajos por cuenta propia sustituyen los empleos formales, en las ciudades y poblados que no tienen grandes números de habitantes y que los primeros no son causa de los otros. Además se puede decir no existe claridad para determinar correlaciones, entre la trilogía antes definida, tasa de desempleo, empleos por cuenta propia y microempresas.

Por lo tanto la discusión se puede centrar en al menos dos flancos, el primero: los trabajos por cuenta propia no son trabajos de "buena calidad", por la informalidad que representan para quienes los practican en materia de tributaria y previsional, no obstante pareciera que disminuyen la tasa de desempleo en la
Región del Maule, a nivel agregado.

En segundo lugar, no se sabe con certeza, si estos trabajos por cuenta propia se convierten en emprendimientos y las estadísticas oficiales actuales, no son capaces de reflejar el fin de estos a lo largo del tiempo. Otro elemento importante a destacar, es que cuando se observan las estadísticas por las ciudades más importantes del Maule, queda al descubierto que son más las microempresas, que los trabajadores por cuenta propia, esto hace prever entonces que los trabajos por cuenta propia, afloran en las ciudades y conglomerados más pequeños de la Región, que en los grandes polos urbanos.

Respecto del emprendimiento no existe evidencia que señale, que más trabajos por cuenta propia, provoquen más emprendimientos en el largo plazo, o inversamente, mientras más emprendimientos menos trabajos por cuenta propia, abriéndose a la discusión que el emprendimiento formal en la Región del Maule, puede estar sujeto a factores socioculturales de quienes los realizan.

Este estudio de carácter descriptivo, es el aliciente para nuevos trabajos de investigación en la materia, puesto que sería interesante entender como los trabajos por cuenta propia, van asociados al nivel socioeconómico, a las conexiones sociales y el nivel educacional de quién los realiza en la Región del Maule.

Por otro lado sería interesante, incluir alguna pregunta en la Encuesta Nacional de Empleo, que busque determinar la continuidad de los trabajos por cuentas propia, de manera de conocer la correlación de estos con la creación de nuevas empresas, con la finalidad de entender la transitoriedad en los empleos por cuenta propia.

Asimismo, es recomendable entender si los 
trabajos por cuenta propia nacen como respuesta a los vaivenes de la economía, es decir, proliferan en periodos de bajo crecimiento económico como empleos de sobrevivencia y que luego decaen, o por el contrario, van de la mano del mayor crecimiento en las regiones y en las grandes ciudades del país. Además es importante señalar que, el concepto por cuenta propia, debería formalizarse tanto en instituciones públicas como privadas, evitando así confundirse con el concepto con microemprendimiento.

De acuerdo a lo encontrado, el comportamiento general de la tasa de desempleo en la Región del Maule, es oscilante, con caídas de la tasa de desempleo en los veranos y aumento de esta en los otoños e inviernos, un patrón similar presentan las tasas de desocupación de las ciudades analizadas; Talca es una ciudad de servicios, que también muestra dicha oscilación, no obstante la caída de las tasas de desempleo no necesariamente se presentan en periodos estivales.

Por otro lado, entre los años 2010-2016, en la Región del Maule, los empleos por cuenta propia muestran un comportamiento inverso a la tasa de desempleo, lo que quiere decir, que cuando una serie aumenta, la otra disminuye. Lo que no se puede saber con certeza, son las relaciones de causalidad entre ambos, tampoco queda muy claro si los empleos por cuenta propia son el precedente de un micro emprendimiento o un emprendimiento.

Finalmente, al analizar los empleos por cuenta propia, la tasa de desempleo y la creación de microempresas al nivel de las ciudades más representativas de la región, se llega a la conclusión general, que no hay claras correlaciones, y que el comportamiento de los empleos por cuenta propia, es diferente en las ciudades grandes respecto de las pequeñas, y que los empleos por cuenta propia en el Maule, son aportados por los pequeños conglomerados urbanos.

Finalmente, a nivel agregado, si pareciera que los trabajos por cuenta propia disminuyen la tasa de desempleo, según lo evidencia la literatura, y el Gráfico $N^{\circ} 1$, no obstante este patrón no es claro en las ciudades más grandes del Maule. Además no se pueden determinar correlaciones entre los trabajos por cuenta propia, desempleo y creación de empresas, en el Maule, en las estadísticas agregadas regionales, ni en las ciudades analizadas. Sin embargo, la importancia de este trabajo radica, en que abre fuentes de investigación futuras, a nivel de regiones, en las tres variables aquí estudiadas.

\section{Bibliografía}

Alvarez, C., Amorós, J. E. y Urbano, D. (2014): Regulations and Entrepreneurship: Evidence from Developed and Developing Countries. INNOVAR Journal, pp. 81-89.

Amorós, J. E., Atienza, M. y Romaní, G. (2008): Formal and Informal Equity Funding in Chile. Estudios de Economía Vol. 35, pp. 179-194.

Austin, J., Stevenson, H. y Wei-Skillern, J. (2006): Social and comercial entrepreneurship: same,different, or both? Entrepeneurship: Theory and Practice Journal, pp. 1-22.

Barletta, F., Robert, V. y Yoguel, G. (2014): Tópicos de la teoría evolucionista neoschumpeteriana de la innovación y el cambio tecnológico. Buenos Aires: Miño y Dávila Editores y Universidad Nacional de General Sarmieto.

Barrera, V. G. (2018): Innovate Self-concept of Micro- 
entrepeneurs: Perception of barriers and Intention to Invest. Brazilian Administration Review, pp. 1-23.

Bellisario, A. (2013): El fin del antiguo régimen agrario chileno. Revista Mexicana de Sociología, 75(3).

Bernal, C. A. (2010): Metodología de la Investigación. Colombia: PEARSON EDUCACION. Biblioteca del Congreso Nacional de Chile (s.f.): https://www.bcn.cl. Recuperado el 28 de Diciembre de 2018, de https:// www.bcn.cl/siit/nuestropais/region7

Castellani, F. y Lora, E. (2014): Is Entrepreneurship a Channel of Social Mobility in Latin America? Latin América Journal Economics, pp. 179-194.

Censo (Diciembre de 2017): Recuperado el 7 de Junio de 2018, de http://www.censo2017.cl/

Fernandez-Serrano, J. y Liñan, F. (2014): Cultura and Entrepeneurship: The Case of Latin America. Revista INNOVAR journal, pp. 169-180.

Instituto Nacional de Estadísticas. (2007): www. censoagropecuario.cl. Recuperado el Diciembre de 2018, de http://www.censoagropecuario.cl/ noticias/07/11/13112007.html

Instituto Nacional de Estadísticas. (2018): Diccionario de Variables Bases de Datos

Encuesta Nacional de Empleo. Santiago: Instituto Nacional de Estadísticas.

Instituto Nacional de Estadísticas. (2018): Manual de usuarios base de datos full. V Encuesta de microemprendimiento EME 2017.

Instituto Nacional de Estadísticas INE. (2010): INE. Recuperado el 7 de Junio de 2018, de http://www. ine.cl/estadisticas/laborales/ene/base-de-datos
Jiménez, R. D. y Páez, C. J. (27 de Junio de 2014): http://www.redalyc.org. Recuperado el 2015 de Marzo de 2018, de http://www.redalyc.org/ html/996/99631890006/

Klein, P. G. (2016): Why entrepeneurs need firms, and the theory of the firm needs entrepeneurship theory. Revista de Administración, pp. 323-326.

Mandakivic, V. y Serey, T. (2017): Reporte Nacional de Chile 2017. Global Entrepeneurship Monitor GEM. Santiago: Gráfica Andes.

Merigó, J. M. y Peris-Ortiz, M. (2014):Entrepreneurship and Decision-Making in Latin America. Revista Innovar Journal, pp. 101-111.

Montero, A. A., Stiepovic, O. P. y Pizarro, V. M. (2015): Global Entrepeneurship Monitor Mujer. Global Entrepeneurship Monito-UDD. Santiago: Donnebaum.

Moyano, D. E., Castillo, G. R. y Lizana, L. J. (2008): Trabajo Informal: Motivos, Bienestar Subjetivo, Salud, y Felicidad en Vendedores Ambulantes. Psicologia em Estudo, Maringá, pp. 693-701.

Peticará, M. y Celhay, P. (Junio de 2010): Informalidad Laboral y Políticas Públicas en Chile- Universidad Alberto Hurtado. Recuperado el 6 de Junio de 2018, de Ideas. Repec: https://ideas.repec.org/p/ila/ilades/ inv257.html

Rodríguez, R. A. (2009): Nuevas perspectivas para entender el emprendimiento empresarial. Pensamiento y Gestión, pp. 94-119.

Servicio de Impuestos Internos SII. (Septiembre de 2016): SIl. Recuperado el 3 de Marzo de 2018, de http://www.sii.cl/estadisticas/empresas.htm 
Subsecretaria de Previsión Social. (2018): https:// www.previsionsocial.gob.cl. Recuperado el 15 de Marzo de 2018, de https://www.previsionsocial.gob. cl/sps/personas/soy-trabajador/soy-independiente/ por-cuenta-propia/

Superintendencia de Bancos e Instituciones Financieras SBIF. (s.f.): Banca fácil. Recuperado el 7 de Junio de 2018, de http://www.bancafacil.cl/ bancafacil/servlet/

\section{Notas}

1. Unidad de Fomento: Unidad de medida que representa la reajustabilidad del peso chileno, de acuerdo con la inflación (Superintendencia de Bancos e Instituciones Financieras SBIF, s.f.).

Recibido: 09 de julio de 2018

Aceptado: 03 de enero de 2019 